\title{
On-site Detection of AHPND in Shrimp Farming by Probe-based Recombinase Polymerase Amplification and Lateral Flow Strip
}

\author{
Xiaohan Yang ${ }^{1}$, Dong Yu ${ }^{1}$, Panpan Zhao ${ }^{2}$, Xin Shen $^{1}$, Ge Jiang ${ }^{3}$, Shiqi Chen ${ }^{1}$, Jingquan \\ Dong $^{1}$, Hui Shen ${ }^{3}$, and Song Gao ${ }^{1}$ \\ ${ }^{1}$ Jiangsu Ocean University \\ ${ }^{2}$ Jilin University \\ ${ }^{3}$ Institute of Oceanology and Marine Fisheries, Jiangsu
}

June 1, 2020

\begin{abstract}
Acute hepatopancreatic necrosis disease (AHPND) is an important bacterial disease occurring early after stocking shrimp fry in shrimp ponds with the mortalities of $100 \%$. AHPND leads to significantly drop in production and brings out huge economic losses worldwide. Thus, rapid, accurate, and convenient on-site detection method is urgent need to monitor the outbreak and spreading of AHPND especially for equipment-poor areas. Application of traditional PCR-based methods is restricted due to the dependence on laboratory equipment and technicians. In this study, an improved isothermal recombinase polymerase amplification (RPA) combined lateral flow strip (LFS) assay was developed for AHPND detection by introducing a probe. The specific primers and probe were designed based on the PirAB gene, chemical modifications were labelled to improve the specificity, and mismatched bases were made to eliminate primer-dependent artifacts. In combination with crude DNA extraction by boiling for $10 \mathrm{~min}$, the RPA-LFS assay could be finished within $25 \mathrm{~min}$ at $37-45^{\circ} \mathrm{C}$ and results were readable by naked eyes. The exclusivity was validated to be no cross-reactivity with 10 other common vibrio spp strains. The inclusivity was verified using 10 other VPAHPND strains isolated from infected shrimps. The limit of detection was 101 colony forming unit $(\mathrm{CFU}) / \mathrm{mL}$ or 102 copies/ $\mu \mathrm{L}$ and $100 \mathrm{CFU} / 10 \mathrm{~g}$ after 2 hours enrichment in spiked shrimp samples. The detection accuracy was evaluated in a total of 75 collected shrimp and seawater samples, which was proven to be consistent with AP4. The established RPA-LFS method provides a rapid, accurate, sensitive and equipment-free approach for on-site detection of AHPND and technical references for monitoring other pathogens in cultivation industry.
\end{abstract}

\section{On-site Detection of AHPND in Shrimp Farming by Probe-based Recombinase Polymerase Amplification and Lateral Flow Strip}

\section{Running title: On-site Detection of AHPND in Shrimps using RPA -LFS}

Xiaohan Yang ${ }^{1,+}$, Yu Dong ${ }^{1,+}$, Panpan Zhao ${ }^{2,+}$, Xin Shen ${ }^{1}$, Ge Jiang ${ }^{3}$, Shiqi Chen ${ }^{1}$, Jingquan Dong1, ${ }^{1}$, Hui $\mathrm{Shen}^{3, *}$, Song Gao ${ }^{4, *}$

${ }^{1}$ Jiangsu Key Laboratory of Marine Biological Resources and Environment, Jiangsu Key Laboratory of Marine Pharmaceutical Compound Screening, Co-Innovation Center of Jiangsu Marine Bio-industry Technology, Jiangsu Ocean University, Lianyungang 222005, China;

${ }^{2}$ Key Laboratory of Zoonosis Research by Ministry of Education, College of Veterinary Medicine, Jilin University, Changchun 130062, China;

${ }^{3}$ Institute of Oceanology and Marine Fisheries, Jiangsu, Jiangsu 226007, China;

${ }^{4}$ School of Pharmacy, Jiangsu Ocean University, Lianyungang 222005, China. 
${ }^{+}$These authors contributed equally to this paper.

${ }^{*}$ Correspondence:

Jingquan Dong

2018000029@jou.edu.cn

Song Gao

2013000002@jou.edu.cn

Hui Shen

darkhui@163.com

\section{Summary}

Acute hepatopancreatic necrosis disease (AHPND) is an important bacterial disease occurring early after stocking shrimp fry in shrimp ponds with the mortalities of $100 \%$. AHPND leads to significantly drop in production and brings out huge economic losses worldwide. Thus, rapid, accurate, and convenient on-site detection method is urgent need to monitor the outbreak and spreading of AHPND especially for equipmentpoor areas. Application of traditional PCR-based methods is restricted due to the dependence on laboratory equipment and technicians. In this study, an improved isothermal recombinase polymerase amplification (RPA) combined lateral flow strip (LFS) assay was developed for AHPND detection by introducing a probe. The specific primers and probe were designed based on the PirAB gene, chemical modifications were labelled to improve the specificity, and mismatched bases were made to eliminate primer-dependent artifacts. In combination with crude DNA extraction by boiling for $10 \mathrm{~min}$, the RPA-LFS assay could be finished within $25 \mathrm{~min}$ at $37-45^{\circ} \mathrm{C}$ and results were readable by naked eyes. The exclusivity was validated to be no crossreactivity with 10 other commonvibrio spp strains. The inclusivity was verified using 10 other $V P_{A H P N D}$ strains isolated from infected shrimps. The limit of detection was $10^{1}$ colony forming unit $(\mathrm{CFU}) / \mathrm{mL}$ or $10^{2}$ copies $/ \mu \mathrm{L}$ and $10^{0} \mathrm{CFU} / 10 \mathrm{~g}$ after 2 hours enrichment in spiked shrimp samples. The detection accuracy was evaluated in a total of 75 collected shrimp and seawater samples, which was proven to be consistent with AP4. The established RPA-LFS method provides a rapid, accurate, sensitive and equipment-free approach for on-site detection of AHPND and technical references for monitoring other pathogens in cultivation industry.

Keywords : Acute hepatopancreatic necrosis disease, shrimp, isothermal recombinase polymerase amplification, lateral flow strip, on-site detection.

\section{Introduction}

In China, shrimp cultivation industry brings out billions of dollars income annually. However, widespread epidemic serious disease of acute hepatopancreatic necrosis disease (AHPND) gives great threat to the shrimp cultivation industry recent years especially for P. monodon, $P$. vannamei, and P. sinensis (Lai et al., 2015). AHPND, also called early mortality syndrome (EMS), usually occurs at the early stage (within 40 days) when shrimp fry is released into shrimp ponds and infected shrimps will probably die in 5 days. Several bacterial pathogens, including Vibrio Parahemolyticus, Vibrio ovensii, Vibrio candida and Vibrio harveyi , are isolated from AHPND infected shrimp (X. Dong et al., 2017; Liu et al., 2015; Tran et al., 2013). Among them, Vibrio Parahemolyticus is verified to be the main cause for AHPND breakout in shrimp cultivation industry. It could produce virulence factors of ToxA and ToxB proteins encoded by PirA-like gene and PirB-like gene, which are located at a pVA1 plasmid(Lee et al., 2015) (X. Dong et al., 2019; $\mathrm{Ng}$ et al., 2018). Shrimps suffering from AHPND mainly manifest slow growth, loss of appetite, lethargy, paleness, soft exoskeletons and atrophy of the hepatopancreas accompanying with empty or discontinuous intestines(Lai et al., 2015). The high mortalities of $100 \%$ caused by AHPND leads to $20 \%$ drops in shrimp production and brings out huge economic losses(Han, Tang, \& Lightner, 2015; Soto-Rodriguez, Gomez-Gil, Lozano-Olvera, Betancourt-Lozano, \& Morales-Covarrubias, 2015). Therefore, a rapid, accurate, and userfriendly on-site detection method for AHPND is essential to monitor its outbreak and spreading in shrimp 
cultivation industry especially for resource-limited areas.

Molecular diagnostic techniques are widely used in pathogen detection due to their specificity and sensitivity (Pang et al., 2019; Saingam, Li, \& Yan, 2018; Xu, Ji, Wu, Yan, \& Chen, 2018). A wide variety of polymerase chain reaction (PCR)-based technologies, including traditional PCR, nested PCR and quantitative real-time PCR (qPCR), have been developed for AHPND detection(Arunrut et al., 2016; Nunan, Lightner, Pantoja, \& Gomez-Jimenez, 2014; Qiu et al., 2018). However, these detection methods require precise temperaturecontrolled equipment, gel electrophoresis, imaging systems or melt-curve analysis, which are limited to laboratory testing and not applied to on-site detection. Resource-limited areas have to look for testing institutions to perform pathogen detection, which is time-causing and takes manpower. To overcome these shortcomings, isothermal amplification techniques are more and more widely used in the field testing, which employ a uniform temperature to amplify targeted nucleic acid sequences and reduce the dependence on thermal cycle machine(Zhao, Chen, Li, Wang, \& Fan, 2015).

The recombinase polymerase amplification (RPA) technology has attracted extensive attention since it could be performed under isothermal conditions of $37^{\circ} \mathrm{C} \sim 45^{\circ} \mathrm{C}$ even body-heat(Y. Dong et al., 2020; Piepenburg, Williams, Stemple, \& Armes, 2006; L. Wang et al., 2019). The RPA technology uses a recombinase to form a nucleoprotein complex with oligonucleotide primers and facilitates the insertion of primers into their complemented double stranded DNA. And then, the extension process is performed by a DNA polymerase at a temperature of $37^{\circ} \mathrm{C}-45^{\circ} \mathrm{C}$. RPA amplification products can be detected by various methods, including agarose gel electrophoresis, fluorescence analysis, and lateral flow test strip (LFS) (J. Wang, Wang, Geng, \& Yuan, 2017; L. Wang et al., 2019; Yang et al., 2018). Among them, the LFS technology is combined with colloidal gold enabling chemically modified DNA molecules to be quickly and visually detected, which eliminates the restricted of laboratory equipment (Miao et al., 2019).

In the RPA-LFS detection system, the 5' end of the forward primer is labeled with FITC, and the 3' end of the reverse primer is labeled with biotin. Therefore, the amplification product is modified with FITC and biotin at the two ends, respectively, and the mouse anti-FITC antibody can capture AuNPs. After the amplification products are loaded onto the sample pad, they migrate through the conjugate pad and are bound with the anti-FITC AuNPs. When these amplification products reach the test line that coated with streptavidin, they are trapped because of the biotin modification and lead to aggregation of AuNPs to show the red color at the test line. The anti-FITC antibody molecules not bound to the amplification product continue migration to the control line that is coated with anti-mouse antibody and aggregate there to validate the strip test. The LFS test enables visual reading of the RPA amplification products with the positive signal as the red band at the test line and the validation signal as the second red band at the control line. The combination of RPA and LFS can further simplify the detection method which makes the detection free from the need for laboratory equipment. However, it is difficult for RPA-LFS system to eliminate the false positive caused by primer-dimers. Measures need to be taken to deal with this critical problem and break out its limitations in application. To solve this problem, we attempted to introduce a specific probe labeled with FITC to reduce the risk of false positive signal. Moreover, the formation of cross dimers caused by probe-reversed primer was analyzed using primer primer 5.0, and the mismatched bases were introduced to eliminate the cross dimers which caused false positive completely.

In this study, we developed a probe-based RPA-LFS system for on-site rapid and visual detection of AHPND. The whole process could be finished within $25 \mathrm{~min}$ at $37^{\circ} \mathrm{C}-45^{\circ} \mathrm{C}$ and presented good specificity and sensitivity. The sensitivity was $10^{1} \mathrm{CFU} / \mathrm{mL}$ or $10^{2}$ copies/reaction and gave a good compatibility with crude template. Moreover, there was no cross-reaction with $V P_{A H P N D \text {-free }}$ and other commonvibrio s. For the detection of low-concentration samples, samples with a concentration of $10^{0} \mathrm{CFU} / 10 \mathrm{~g}$ could be detected with a shorter enrichment time. This RPA-LFS assay would benefit the on-site AHPND detection in resourcelimited conditions for aquaculture of shrimp.

\section{Method}

\subsection{Bacteria strains and culture conditions}


All strains used in this study are listed in Table 1 . Among them, AHPND pathogenic Vibrio. Parahaemolyticus $\left(V P_{A H P N D}\right)$, Vibrio. parahaemolyticus(V. parahaemolyticus), Vibrio vulnificus (V .vulnificus), Vibrio alginolyticus ( $V$.alginolyticus ), Vibrio cholerae ( $V$. cholerae), Vibrio harveyi( $V$. harveyi ), Vibrio mediterranei (V. mediterranei), Vibrio shilonii( $V$. shilonii ) were obtained from Institute of Oceanology and Marine Fisheries (Nantong, China). Vibrio splendidus (V. splendidus), Vibrio mimicus (V. mimicus ), Vibrio(ichthyoenteri ), V. ichthyoenteri were purchased from Marine Culture of China. All strains were identified using individually recommended gold standard. All strains were inoculated into $3 \% \mathrm{NaCl}$ alkaline protein water (APW) and cultured according to different conditions of bacterial.

\subsection{DNA template preparation}

Crude genomic DNA from each pure reference stain culture was extracted by boiling for 10 min. For spiked samples, $1 \mathrm{~mL}$ of culture solution were centrifugated at $800 \times \mathrm{g}$ for $10 \mathrm{~min}$ to remove the tissue and the supernatant was then centrifugated at $10,000 \times \mathrm{g}$ for $10 \mathrm{~min}$. Pellets were collected and resuspended using $200 \mu \mathrm{L}$ PBS. DNA was released after boiling and used as template for RPA-LFS detection. For collected raw shrimps, genomic DNA was extracted using TIANamp Genomic DNA Kit (TIANGEN BIOTECH CO.,LTD., Beijing, China) according to the manufacture's protocol. The extracted genomic DNA was stored at $-80^{\circ} \mathrm{C}$.

\subsection{Primer BLAST}

In order to screen specific and sensitive primers for detecting AHPND, primers were designed based on the conserved sequences of PirAB gene in $V P_{-A H P N D}$ (GenBank \# U50548.1) using NCBI Primer-BLAST (https://www.ncbi.nlm.nih.gov/tools/primer-blast). The product size was set as min at 200 and max at 500. Tm value was set at 20-80. To ensure the specificity of the primers, the species was restricted to $V$. parahaemolyticus. The maximal self-complementarity was set as any at 4 and 3' at 1 . The maximal pair complementarity was set as any at 4 and 3' at 1 . Other parameters were set as default. Six pairs of primers were selected as candidates for sensitivity test (Table. 2 ).

\subsection{RPA procedure and electrophoresis}

RPA reactions were carried out according to the manufacturer's protocol of TwistAmp ${ }^{\circledR}$ Liquid DNA Amplification Kit (TwistDx Inc., Maidenhead, UK). Each $50 \mu \mathrm{L}$ reaction system contained $25 \mu \mathrm{L}$ of $2 \times$ Reaction buffer, $5 \mu \mathrm{L}$ of $10 \times$ Basic e-mix, $2.5 \mu \mathrm{L}$ of $20 \times$ core mix, $2.1 \mu \mathrm{L}$ of each primer $(10 \mu \mathrm{M}$, General Biosystems Co. Ltd, Anhui, China), and $9.8 \mu \mathrm{L}$ of distilled water. After vortexing and spinning briefly, $1 \mu \mathrm{L}$ of template and $2.5 \mu \mathrm{L}$ of magnesium acetate $(280 \mathrm{mM})$ were added to the lid ensuring all the components reacted at the same time. The reaction mixture was immediately incubated at $37^{\circ} \mathrm{C}$ for $30 \mathrm{~min}$. The products of RPA were purified using PCR Cleaning Kit (Monad Biotech Co., Ltd, Wuhan, China) and electrophoresed on a $1.5 \%$ agarose gel.

\subsection{Design of probes}

The probes used in RPA-LFS assay were designed using Primer Premier 5 software. The target sequence of the selected primers was entered. The size of the probe was set at $46 \mathrm{bp} \sim 52 \mathrm{bp}$. The Tm value was set at 50-100. The GC content was set at 20-70. The max hairpin score was set as 9 . The max primer-dimer score was set as 9 . The max poly-X was set as 5 . Other parameters were set as default. Probe sequences were listed in Table. 3 .

\subsection{Mismatches between probe and reverse primer}

The weaker false-positive signal formed by the reverse primer and probe was analyzed using primer primer 5.0 software. To eliminate the possible cross dimer, places where may probably lead to false signals were picked out, base mismatches were made to break continuous paired bases and prevented the formation of primer and probe complexes. Thus, false signals were eliminated, ensuring the accuracy of RPA-LFS method. Modified primers and probes were listed in Table. 4 .

\subsection{RPA-LFS procedure}


The RPA-LFS assay was performed according to the manufacturer's protocol of TwistAmp ${ }^{\circledR}$ DNA Amplification nfo Kit (TwistDx). Each $50 \mu \mathrm{L}$ reaction system contained $29.5 \mu \mathrm{L}$ of Rehydration Buffer, $2.1 \mu \mathrm{L}$ of each primer $(10 \mu \mathrm{M}), 0.6 \mu \mathrm{L}$ of probe $(10 \mu \mathrm{M})$, and $12.2 \mu \mathrm{L}$ of distilled water. To initiate the reaction, $1 \mu \mathrm{L}$ of template and $2.5 \mu \mathrm{L}$ of magnesium acetate $(280 \mathrm{mM})$ were added to the lid. After vortexing and spinning briefly, the reaction mixture was incubated at the corresponding time and temperature conditions. Amplification products were detected by LFS (Ustar Biotechnologies Ltd, Hangzhou, China). Five microliter of amplification products was added into a $1.5 \mathrm{~mL}$ centrifuge tube containing $95 \mu \mathrm{L}$ buffer (Ustar Biotechnologies), the stick of LFS was then inserted into the tube, and the result could be read visually.

\subsection{Sensitivity of probe-based RPA-LFS method}

To determine the limit of detection (LOD), tenfold gradient dilutions of pure cultures $\left(10^{7}-10^{1} \mathrm{CFU} / \mathrm{mL}\right)$ and purified pVA1 plasmid $\left(10^{7}-10^{1}\right.$ copies $\left./ \mu \mathrm{L}\right)$ isolated from $V P_{A H P N D}$ were prepared and detected. Meanwhile, shrimps purchased from the local market, were verified to be $V P_{A H P N D}$ free using AP4 methods described as below. One gram of shrimp hepatopancreas was homogenized using $1 \mathrm{~mL}$ of $V P_{A H P N D}$ pure cultures $\left(10^{8}\right.$ $\left.10^{2} \mathrm{CFU} / \mathrm{mL}\right)$ and $1 \mathrm{~mL}$ of purified pVA1 plasmid $\left(10^{8}-10^{1}\right.$ copies $\left./ \mu \mathrm{L}\right)$, respectively. The spiked homogenates were added to $9 \mathrm{~mL}$ of APW and shaken at $200 \mathrm{rpm}$ for $1 \mathrm{~min}$. One milliliter of spiked culture was collected and tested by RPA-LFS. For lower contaminated situations, pre-enrichment was carried out. In detail, shrimps purchased from the local market were treated with alcohol and verified to be $V P_{A H P N D}$ free using AP4 method. Ten gram of tissue samples were weighted, homogenized in $9 \mathrm{~mL}$ of APW, and spiked with $1 \mathrm{~mL}$ of $V P_{A H P N D}$ to a final concentration ranging from $10^{2} \mathrm{CFU} / 10 \mathrm{~g}$ to $10^{0} \mathrm{CFU} / 10 \mathrm{~g}$. The mixture was then cultured at $37^{\circ} \mathrm{C}$ with shaking at $200 \mathrm{rpm}$. After $0,2,4,6,8,12$ and 24 hours treatment, $1 \mathrm{~mL}$ of culture solution was collected and tested using RPA-LFS.

\subsection{AP4 assay}

The DNA extracted from bacterial samples were used as templates for two-tube nested PCR method (AP4). The procedure of AP4 was carried out referring to previous described report(Arunrut et al., 2016). Two pairs of specific primers (AP4-F1: 5'-ATGAGTAACAATATAAAACATGAAAC-3'; AP4-R1: 5'-ACGATTTCGACGTTCCCCAA-3' and AP4-F2: 5'-TTGAGAATACGGGACGTGGG-3'; AP4-R2: 5'GTTAGTCATGTGAGCACCTTC-3') targeting to the PirA gene of $V P_{A H P N D}$ was used for AP4 detection. The first step was performed contained the following components: $25 \mu \mathrm{L}$ of MonAmp $2 \times$ Taq Mix (+Dye), $1 \mu \mathrm{L}$ of AP4-F1 $(10 \mu \mathrm{M}), 1 \mu \mathrm{L}$ of AP4-R1 $(10 \mu \mathrm{M}), 1 \mu \mathrm{L}$ of the template and $21 \mu \mathrm{L}$ of $\mathrm{ddH}_{2} \mathrm{O}$. Initial denaturation at $95^{\circ} \mathrm{C}$ for $30 \mathrm{~s}$ followed by 30 cycles of denaturation at $95^{\circ} \mathrm{C}$ for $20 \mathrm{~s}$, primer annealing at $55^{\circ} \mathrm{C}$ for $20 \mathrm{~s}$ and elongation at $72^{\circ} \mathrm{C}$ for $20 \mathrm{~s}$. The PCR products were used as template for the second step PCR amplification, which contained the following components: $25 \mu \mathrm{L}$ of MonAmp $2 \times$ Taq Mix (+Dye), $1 \mu \mathrm{L}$ of AP4-F2 $(10 \mu \mathrm{M}), 1 \mu \mathrm{L}$ of AP4-R2 $(10 \mu \mathrm{M}), 1 \mu \mathrm{L}$ of forward and reverse primer $(10 \mu \mathrm{M}), 1 \mu \mathrm{L}$ of the first step products and $21 \mu \mathrm{L}$ of $\mathrm{ddH}_{2} \mathrm{O}$. Initial denaturation at $95^{\circ} \mathrm{C}$ for $30 \mathrm{~s}$ followed by 25 cycles of denaturation at $95^{\circ} \mathrm{C}$ for $20 \mathrm{~s}$, primer annealing at $55^{\circ} \mathrm{C}$ for $20 \mathrm{~s}$ and elongation at $72^{\circ} \mathrm{C}$ for $20 \mathrm{~s}$. The second step products were used for agarose gel electrophoresis detection.

\subsection{Evaluation of RPA-LFS in clinical samples}

The feasibility of probe-based RPA-LFS assay was evaluated in 65 clinical shrimp samples and 10 collected seawater samples. After a disinfection treatment with ethanol, $10 \mathrm{mg}$ tissue of shrimp samples was weighted, cut into small pieces, and genomic DNA was extracted with TIANamp Genomic DNA kit (Tiangen Biotech Co. Ltd) dissolving in $50 \mu \mathrm{L}$ elution buffer. For clinical water genomic DNA extraction, $5 \mathrm{~mL}$ of samples were centrifuged at $10,000 \times \mathrm{g}$ for $30 \mathrm{~min}$, washed twice with $\mathrm{ddH}_{2} \mathrm{O}$, and then resuspended using $100 \mu \mathrm{L}$ $\mathrm{ddH}_{2} \mathrm{O}$. One microlitre of purified genomic DNA was used as the template for RPA-LFS. AP4 method was used as control.

\section{Results}

\subsection{Screening of AHPND detection primers}

Rational design of primer pairs for AHPND detection started with a primer BLAST searching on the se- 
quences of virulence genes of $V P_{A H P N D}$, PirAB. RPA assay was performed to screen the amplification ability and species specificity of the designed six primer pairs. Results showed that all the primer pairs gave clear and specific bands, but primer pairs of $V P_{A H P N D}-2$ and $V P_{A H P N D}-4$ also had primer-dimer bands in the no template control (NTC) group and primer pairs of $V P_{A H P N D}-5$ and $V P_{A H P N D}-6$ displayed weak bands. Compared with other 4 pairs of primers, $V P_{A H P N D}-1$ and $V P_{A H P N D}-3$ showed good amplification performance without primer-dimer bands (Fig. 1 ). Thus, primer pairs of $V P_{A H P N D}-1$ and $V P_{A H P N D}-3$ were selected as candidates for RPA detection of AHPND.

The specificity of primer pairs of $V P_{A H P N D}-1$ and $V P_{A H P N D}-3$ were measured using genomic DNA of $V P_{A H P N D}, V P_{A H P N D}$-free and 9 other pathogenic bacterial species that were commonly existed in the mariculture, including $V$. vulnificus, $V$. alginolyticus, $V$. cholerae, $V$. harveyi, $V$. mediterranei, $V$. shilonii, V. splendidus, V. mimicus, V. ichthyoenteri. Positive result was observed only in the template of $V P_{A H P N D}$ while no amplification bands occurred when using all the other species. Taken together, primer pairs of $V P_{A H P N D}-1$ and $V P_{A H P N D}-3$ were highly specific for the identification of AHPND (Fig. 2 ).

\subsection{Performance of primer-probesets}

In order to improve the RPA assay to achieve equipment-free dependence, specific probes were designed targeting to the selected primer pairs of $V P_{A H P N D}-1$ and $V P_{A H P N D}-3$. Probes were located between each forward primer and reverse primer. RPA-LFS assay was performed to evaluate the feasibility of primer-probe sets. Results showed that both primer-probe sets gave positive signals, that is, both the detection line and the control line had red bands. However, the NTC groups also displayed positive signals at the control lines and false positive signals at the test lines. Moreover, probe1/VPAHPND -1 gave weaker false signal than probe2/ $V P_{A H P N D}-3$, which may be easier to be eliminated (Fig. 3a ).

\subsection{Elimination of false positive signals by modifying bases}

To successfully establish the RPA-LFS assay for AHPND, it was essential to eliminate the false positive signals during detection. To solve this problem of false signals, the cross dimer of probe and reverse primer was analyzed using primer primer 5.0 software. Results showed that three cross dimers which may cause false positive signals were formed. To avoid the formation of cross dimer, base mismatches were introduced between the probe 1 and the reverse primer of $V P_{A H P N D}-1$. One $T$ base on the reverse primer was replaced with an $\mathrm{A}$ base. Two $\mathrm{A}$ bases and a $\mathrm{C}$ base on the probe were replaced with $\mathrm{T}$ and $\mathrm{G}$ bases, respectively. Subsequently, RPA-LFS assay was performed to identify the feasibility of mismatched primer-probe set, including amplification performance and false positive signals. Results showed that the false positive signals were successfully eliminated, and amplification performance were not influenced when using mismatched probe and reverse primer (Fig. $3 \mathbf{b})$.

\subsection{Optimization of the probe-based RPA-LFS conditions}

To optimize the RPA-LFS reaction temperature, the RPA assay was performed at temperatures ranging from $25^{\circ} \mathrm{C}$ to $45^{\circ} \mathrm{C}$. The reaction time was set at $30 \mathrm{~min}$ and the amplification results were analyzed by LFS. As shown in figure 4 , a red visual band at the test line appeared at $37^{\circ} \mathrm{C}$ to $45^{\circ} \mathrm{C}$, but a weak band on test line at $30^{\circ} \mathrm{C}$ (Fig. 4a ).

The RPA reaction time was screened from 5 min to $40 \mathrm{~min}$ at $37^{\circ} \mathrm{C}$. A red band appeared on test line at $10 \mathrm{~min}$ and intensified with the increase of time. After $20 \mathrm{~min}$, the intensity of the band did not change significantly (Fig. 4b ). Therefore, the optimum reaction condition was $37^{\circ} \mathrm{C}$ to $45^{\circ} \mathrm{C}$ and $20 \mathrm{~min}$.

\subsection{Exclusivity and inclusivity analysis of the RPA-LFS assay}

Exclusivity of the optimized probe-based RPA-LFS assay was detected using $V P_{A H P N D}, V P_{A H P N D}$-free and 9 othervibriospecies, including $V$. vulnificus, V. alginolyticus, V. cholerae, V. harveyi, V. mediterranei, V. shilonii, V. splendidus, V. mimicus, and V. ichthyoenteri . Inclusivity of the RPA-LFS assay was tested using the genomic DNA of 10 other $V P_{A H P N D}$ strains isolated from shrimps with AHPND. Results showed that the RPA-LFS assay gave positive signals only in the template of $V P_{A H P N D}$ and 10 other $V P_{A H P N D}$ 
isolated from shrimps with AHPND while negative signals using templates of $V P_{A H P N D}$-free and othervibrio species (Fig. 5 ). These results confirmed the detection specificity of the RPA-LFS assay.

\subsection{LOD determination of the RPA-LFS assay}

The LOD of the RPA-LFS assay was evaluated under various conditions. Firstly, DNA templates from a 10-fold series dilution of purified $V P_{A H P N D}$ culture and interfered with shrimp homogenates to final concentrations ranging from $10^{7} \mathrm{CFU} / \mathrm{mL}$ to $10^{1} \mathrm{CFU} / \mathrm{mL}$ were tested. Results showed that, a red band appeared at the $10^{1} \mathrm{CFU} / \mathrm{mL}$ group either in the pure culture (Fig. 6a ) or spiked samples (Fig. 6b ). And the test lines intensified with the increasing amount of $V P_{A H P N D}$. Secondly, the 10-fold series dilution of purified pVA1 plasmid and interfered with shrimp homogenates to a final concentration ranging from $10^{7} \mathrm{copies} / \mu \mathrm{L}$ to $10^{0}$ copies $/ \mu \mathrm{L}$ were tested to evaluate the sensitivity of RPA-LFS, respectively. Although weak, $10^{2}$ copies $/ \mu \mathrm{L}$ can still be detected either in purified (Fig. 7a ) or spiked samples (Fig. $7 \mathbf{b})$. These results showed that the LOD of RPA-LFS assay was $10^{1} \mathrm{CFU} / \mathrm{mL}$ or $10^{2}$ copies $/ \mu \mathrm{L}$ and the probe-based RPA-LFS system shared good compatibility with food matrix.

\subsection{Detection for per-enrichment spiked samples}

For low concentration sample, one-step enrichment is necessary to reach a detectable concentration for accurate diagnosis. Ten grams of shrimp homogenate were spiked with different amounts of $V P_{A H P N D}$ to final concentrations ranging from $10^{2} \mathrm{CFU} / 10 \mathrm{~g}$ to $1 \mathrm{CFU} / 10 \mathrm{~g}$ and detected after 2-24 hours enrichment. Results showed that all spiked shrimp samples with different concentrations of $V P_{A H P N D}$ were detected after 2 hours enrichment. Moreover, the positive signal intensified along with the increased amounts of spiked cultures and enrichment time (Fig. 8 ). These results implied that RPA-LFS assay combined with 2 hours enrichment time could detect as low as $1 \mathrm{CFU} / 10 \mathrm{~g}$ for spiked samples, which demonstrated great advantages in the diagnosis of AHPND.

\subsection{Evaluation of the probe-based RPA-LFS assay}

To evaluate the accuracy of the RPA- LFS, 65 clinical shrimp samples and 10 seawater samples were tested and compared with the standard method of AP4 simultaneously. Results showed that 20 of 65 shrimp samples and 3 of 10 water samples were AHPND positive, which is consistent with that of the AP4 (Table 5 ). These data indicated that the established probe-based RPA-LFS assay was highly accurate and had excellent application performance.

\section{Discussion}

AHPND is one of the most important diseases that seriously affect the shrimp farming in recent years. The outbreak of this disease will lead to a significant decline in shrimp production in a short period of time, causing serious economic losses(Dhar et al., 2019). Rapid and accurate detection method of AHPND are essential for effective control of its outbreak and spreading. RPA technology is widely used in molecular diagnostics for its fast, portable and equipment-free advantages(Li, Macdonald, \& von Stetten, 2018). LFS technology can quickly detect modified DNA amplification products and simplify the detection procedures of RPA products, which makes the RPA reaction completely eliminate the dependence on laboratory equipment. However, RPA-LFS combined methods are facing problems of false negative signals in its practical application. In this study, we made attempts to solve the critical problem realizing rapid on-site RPA-LFS detection of AHPND.

In molecular diagnosis, false positive signals caused by primer dimers are unavoidable(Meagher, Priye, Light, Huang, \& Wang, 2018; Rodriguez, Rodriguez, Cordoba, \& Andrade, 2015). Unlike nucleic acid electrophoresis, it is impossible for RPA-LFS detection method to distinguish false positive signals caused by primer dimers by analyzing the size of the amplified bands. To overcome this problem, a specific probe was introduced. Previous research reported that the introduction of probes not only increased the specificity of RPA-LFS assay, but also reduced the risk of false positives(Piepenburg et al., 2006). In this study, FITC was labeled on the 5' end of the probe instead of the forward primer, therefore, the generation of primer dimer will not cause false positive signal in the LFS detection. In addition, the amount of probe used in this assay was much less than that of primer, which would significantly reduce the risk of false positives formed 
by the probe and reverse primer. However, we found that the introduction of probes could not completely eliminate false positive signals (Fig. 3a ). Small amount of cross dimer formed by the probe and the reverse primer could be detected by the highly sensitive LFS detection method and exposed a risk of false positives. Thus, strategies should be taken to further deal with this issue.

Previous studies showed that introduction of base mismatches in RPA primers could avoid nonspecific amplification without affecting its amplification performance(Daher, Stewart, Boissinot, Boudreau, \& Bergeron, 2015; Lillis et al., 2016). Therefore, we tried to make base mismatches between the probe and reverse primer to prevent the formation of probe and reverse primer complexes. The formation of cross dimer between the probe and the reverse primer was firstly analyzed using primer primer 5.0 software. Then, continuous paired bases were determined and substituted between the probe and the reverse primer. Results showed that the false positive signals caused by cross dimer was successfully eliminated after introducing mismatched bases (Fig. 3b).

The probe-based RPA-LFS detection method established in this study shared good sensitivity and compatibility with crude samples or food matrix. The RPA-LFS assay was able to detect $10^{1} \mathrm{CFU} / \mathrm{mL}$ crude template from $V P_{A H P N D}$ culture or $10^{2}$ copies/ $\mu \mathrm{L}$ purified pVA1 plasmid. Moreover, the sensitivity of RPALFS assay wasn't affected after mixing $V P_{A H P N D}$ culture or pVA1 plasmid with shrimp tissue homogenates. The LOD of established RPA-LFS system were 10-times more sensitive than nest PCR detection method $\left(10^{2} \mathrm{CFU} / \mathrm{mL}\right)$ and 1000-times more sensitive than one-step PCR detection method $\left(10^{3} \mathrm{CFU} / \mathrm{mL}\right)$ when detecting DNA extracted from shrimps infected with $V P_{A H P N D}$ (Arunrut et al., 2016; Sirintip Dangtip, 2015). For the low concentration template samples, the PCR-based method required one-step overnight enrichment to make the detection of pathogenic bacteria reach the detectable amount, which consumed too much time(Choi et al., 2018). For the established RPA-LFS system, $10^{0} \mathrm{CFU} / 10 \mathrm{~g}$ templates could be detected after $2 \mathrm{~h}$ enrichment time, which significantly shortened the enrichment time of samples and improved the detection efficiency (Fig. 8 ).

To evaluate the detection accuracy of the probe-based RPA-LFS assay, a total of 75 clinical samples, including 65 of shrimp samples and 10 of seawater samples, were collected and detected using AR4 method at the same time. Results showed that 23 of 75 samples gave the positive signal which was consistent with AP4 detection method. These data implied that the RPA-LFS assay shared good feasibility in clinical samples detection and was of great significance in the monitoring of AHPND during shrimp farming.

\section{Conclusions}

In this study, we developed an RPA-LFS assay for rapid on-site detection of AHPND. This method could be completed at $37-45^{\circ} \mathrm{C}$ within 25 min exhibiting good specificity and sensitivity. The LOD was as low as $10^{1}$ $\mathrm{CFU} / \mathrm{mL}, 10^{2}$ copies $/ \mu \mathrm{L}$, and $1 \mathrm{CFU} / 10 \mathrm{~g} V P_{A H P N D}$ spiked samples after $2 \mathrm{~h}$ pre-enrichment. For clinical samples detection, 23 of 75 clinical samples were positive for AHPND, which was consistent with that of AP4. The coincidence rate was 100\% between the improved RPA-LFS and AP4 method. Overall, the RPALFS detection system is rapid, specific, sensitive, portable, simple to be operated, and not dependent on the laboratory and experimental technicians. Thus, it has good development prospects in on-site detection, which is of great significance to prevent and monitor AHPND during the cultivation of shrimps.

\section{Author contributions}

D. J. and Song Gao directed the program. D. J. and Y. X. designed this research. Y. X. performed the experiments. Y. X. and Z.P. wrote this manuscript. Hui Shen and Ge Jiang provided the clinical materials. Xin Shen, Yu Dong and Shiqi Chen analyse the experiment data.

\section{Conflicts of interest}

We have no conflicts of interest regarding the publication of the manuscript.

\section{Acknowledgements}


This work was supported by grants from the National Natural Science Foundation of China (31470275), the Fishery Science and Technology Innovation Program (Y2018-14), the Nantong Municipal Science and Technology Plan (GJZ17077), the Open Foundation of Jiangsu Key Laboratory of Marine Pharmaceutical Compound Screening (HY201805) and the Priority Academic Program Development of Jiangsu Higher Education Institutions of China. The funding body provided monetary support only and did not have any input into the design of the study and collection, analysis, and interpretation of data and in writing the manuscript.

\section{Data availability statement}

The datasets referred in this study are available from the corresponding author upon request.

\section{Ethical Statement - animal}

No animal was sacrificed for the purpose of this study. No separate ethical approval was required for the study.

\section{Reference}

Arunrut, N., Kampeera, J., Sirithammajak, S., Sanguanrut, P., Proespraiwong, P., Suebsing, R., \& Kiatpathomchai, W. (2016). Sensitive Visual Detection of AHPND Bacteria Using Loop-Mediated Isothermal Amplification Combined with DNA-Functionalized Gold Nanoparticles as Probes. PLoS One, 11 (3), e0151769. doi:10.1371/journal.pone.0151769

Choi, Y., Lee, S., Lee, H., Lee, S., Kim, S., Lee, J., . . . Yoon, Y. (2018). Rapid Detection of Escherichia coli in Fresh Foods Using a Combination of Enrichment and PCR Analysis. Korean J Food Sci Anim Resour, 38 (4), 829-834. doi:10.5851/kosfa.2018.e19

Daher, R. K., Stewart, G., Boissinot, M., Boudreau, D. K., \& Bergeron, M. G. (2015). Influence of sequence mismatches on the specificity of recombinase polymerase amplification technology. Mol Cell Probes, 29 (2), 116-121. doi:10.1016/j.mcp.2014.11.005

Dhar, A. K., Piamsomboon, P., Aranguren Caro, L. F., Kanrar, S., Adami, R., Jr., \& Juan, Y. S. (2019). First report of acute hepatopancreatic necrosis disease (AHPND) occurring in the USA. Dis Aquat Organ, 132 (3), 241-247. doi:10.3354/dao03330

Dong, X., Song, J., Chen, J., Bi, D., Wang, W., Ren, Y., . . . Huang, J. (2019). Conjugative Transfer of the pVA1-Type Plasmid Carrying the pirAB (vp) Genes Results in the Formation of New AHPND-Causing Vibrio.Front Cell Infect Microbiol, 9 , 195. doi:10.3389/fcimb.2019.00195

Dong, X., Wang, H., Xie, G., Zou, P., Guo, C., Liang, Y., \& Huang, J. (2017). An isolate of Vibrio campbellii carrying the $\operatorname{pir}(\mathrm{VP})$ gene causes acute hepatopancreatic necrosis disease. Emerg Microbes Infect, 6 (1), e2. doi:10.1038/emi.2016.131

Dong, Y., Zhao, P., Chen, L., Wu, H., Si, X., Shen, X., . . . Gao, S. (2020). Fast, simple and highly specific molecular detection of Vibrio alginolyticus pathogenic strains using a visualized isothermal amplification method. BMC Vet Res, 16 (1), 76. doi:10.1186/s12917-020-02297-4

Han, J. E., Tang, K. F., \& Lightner, D. V. (2015). Genotyping of virulence plasmid from Vibrio parahaemolyticus isolates causing acute hepatopancreatic necrosis disease in shrimp. Dis Aquat Organ, 115 (3), 245-251. doi:10.3354/dao02906

Lai, H. C., Ng, T. H., Ando, M., Lee, C. T., Chen, I. T., Chuang, J. C., . . . Wang, H. C. (2015). Pathogenesis of acute hepatopancreatic necrosis disease (AHPND) in shrimp. Fish Shellfish Immunol, 47 (2), 1006-1014. doi:10.1016/j.fsi.2015.11.008

Lee, C. T., Chen, I. T., Yang, Y. T., Ko, T. P., Huang, Y. T., Huang, J. Y., . . . Lo, C. F. (2015). The opportunistic marine pathogen Vibrio parahaemolyticus becomes virulent by acquiring a plasmid that 
expresses a deadly toxin. Proc Natl Acad Sci U S A, 112 (34), 10798-10803. doi:10.1073/pnas.1503129112

Li, J., Macdonald, J., \& von Stetten, F. (2018). Review: a comprehensive summary of a decade development of the recombinase polymerase amplification. Analyst, 144 (1), 31-67. doi:10.1039/c8an01621f

Lillis, L., Lehman, D. A., Siverson, J. B., Weis, J., Cantera, J., Parker, M., . . . Boyle, D. S. (2016). Cross-subtype detection of HIV-1 using reverse transcription and recombinase polymerase amplification. $J$ Virol Methods, 230 , 28-35. doi:10.1016/j.jviromet.2016.01.010

Liu, L., Xiao, J., Xia, X., Pan, Y., Yan, S., \& Wang, Y. (2015). Draft Genome Sequence of Vibrio owensii Strain SH-14, Which Causes Shrimp Acute Hepatopancreatic Necrosis Disease. Genome Announc, 3 (6). doi:10.1128/genomeA.01395-15

Meagher, R. J., Priye, A., Light, Y. K., Huang, C., \& Wang, E. (2018). Impact of primer dimers and self-amplifying hairpins on reverse transcription loop-mediated isothermal amplification detection of viral RNA. Analyst, 143 (8), 1924-1933. doi:10.1039/c7an01897e

Miao, F., Zhang, J., Li, N., Chen, T., Wang, L., Zhang, F., . . . Hu, R. (2019). Rapid and Sensitive Recombinase Polymerase Amplification Combined With Lateral Flow Strip for Detecting African Swine Fever Virus. Front Microbiol, 10 , 1004. doi:10.3389/fmicb.2019.01004

Ng, T. H., Lu, C. W., Lin, S. S., Chang, C. C., Tran, L. H., Chang, W. C., . . . Wang, H. C. (2018). The Rho signalling pathway mediates the pathogenicity of AHPND-causing V. parahaemolyticus in shrimp. Cell Microbiol, 20 (8), e12849. doi:10.1111/cmi.12849

Nunan, L., Lightner, D., Pantoja, C., \& Gomez-Jimenez, S. (2014). Detection of acute hepatopancreatic necrosis disease (AHPND) in Mexico.Dis Aquat Organ, 111 (1), 81-86. doi:10.3354/dao02776

Pang, B., Yao, S., Xu, K., Wang, J., Song, X., Mu, Y., . . . Li, J. (2019). A novel visual-mixeddye for LAMP and its application in the detection of foodborne pathogens. Anal Biochem, 574, 1-6. doi:10.1016/j.ab.2019.03.002

Piepenburg, O., Williams, C. H., Stemple, D. L., \& Armes, N. A. (2006). DNA detection using recombination proteins. PLoS Biol, 4 (7), e204. doi:10.1371/journal.pbio.0040204

Qiu, L., Chen, M. M., Wan, X. Y., Zhang, Q. L., Li, C., Dong, X., . . . Huang, J. (2018). Detection and quantification of shrimp hemocyte iridescent virus by TaqMan probe based real-time PCR. J Invertebr Pathol, 154 , 95-101. doi:10.1016/j.jip.2018.04.005

Rodriguez, A., Rodriguez, M., Cordoba, J. J., \& Andrade, M. J. (2015). Design of primers and probes for quantitative real-time PCR methods. Methods Mol Biol, 1275 , 31-56. doi:10.1007/978-1-4939-2365-6_3

Saingam, P., Li, B., \& Yan, T. (2018). Use of amplicon sequencing to improve sensitivity in PCRbased detection of microbial pathogen in environmental samples. J Microbiol Methods, 149 , 73-79. doi:10.1016/j.mimet.2018.05.005

Sirintip Dangtip, Ratchanok Sirikharin, Piyachat Sanguanrut (2015). AP4 method for two-tube nested PCR detection of AHPND isolates of Vibrio parahaemolyticus. Aquaculture Reports, (2015), 158-162.

Soto-Rodriguez, S. A., Gomez-Gil, B., Lozano-Olvera, R., Betancourt-Lozano, M., \& Morales-Covarrubias, M. S. (2015). Field and experimental evidence of Vibrio parahaemolyticus as the causative agent of acute hepatopancreatic necrosis disease of cultured shrimp (Litopenaeus vannamei) in Northwestern Mexico. Appl Environ Microbiol, 81 (5), 1689-1699. doi:10.1128/AEM.03610-14

Tran, L., Nunan, L., Redman, R. M., Mohney, L. L., Pantoja, C. R., Fitzsimmons, K., \& Lightner, D. V. (2013). Determination of the infectious nature of the agent of acute hepatopancreatic necrosis syndrome affecting penaeid shrimp. Dis Aquat Organ, 105 (1), 45-55. doi:10.3354/dao02621 
Wang, J., Wang, J., Geng, Y., \& Yuan, W. (2017). A recombinase polymerase amplification-based assay for rapid detection of African swine fever virus. Can J Vet Res, 81 (4), 308-312.

Wang, L., Zhao, P., Si, X., Li, J., Dai, X., Zhang, K., . . . Dong, J. (2019). Rapid and Specific Detection of Listeria monocytogenes With an Isothermal Amplification and Lateral Flow Strip Combined Method That Eliminates False-Positive Signals From Primer-Dimers. Front Microbiol, 10 , 2959. doi:10.3389/fmicb.2019.02959

Xu, D., Ji, L., Wu, X., Yan, W., \& Chen, L. (2018). Detection and differentiation of Vibrio parahaemolyticus by multiplexed real-time PCR.Can J Microbiol, 64 (11), 809-815. doi:10.1139/cjm-2018-0083

Yang, H. L., Wei, S., Gooneratne, R., Mutukumira, A. N., Ma, X. J., Tang, S. Z., \& Wu, X. Y. (2018). Development of a recombinase polymerase amplification assay for Vibrio parahaemolyticus detection with an internal amplification control. Can J Microbiol, 64 (4), 223-230. doi:10.1139/cjm-2017-0504

Zhao, Y., Chen, F., Li, Q., Wang, L., \& Fan, C. (2015). Isothermal Amplification of Nucleic Acids. Chem Rev, 115 (22), 12491-12545. doi:10.1021/acs.chemrev.5b00428

Table 1 Information of bacteria strains used in the establishment of the RPA-LFS assay.

\begin{tabular}{ll}
\hline Name & Source \\
\hline$V P_{A H P N D}$ & Nantong, China \\
$V P_{A H P N D}$ & Nantong, China \\
$V P_{A H P N D}$ & Nantong, China \\
$V P_{A H P N D}$ & Nantong, China \\
$V P_{A H P N D}$ & Nantong, China \\
$V P_{A H P N D}$ & Nantong, China \\
$V P_{A H P N D}$ & Nantong, China \\
$V P_{A H P N D}$ & Nantong, China \\
$V P_{A H P N D}$ & Nantong, China \\
$V P_{A H P N D}$ & Nantong, China \\
$V P_{A H P N D}$ & Nantong, China \\
$V P_{A H P N D-f r e e}$ & ATCC 17802 \\
$V$. vulnificus & ATCC 27562 \\
$V$. alginolyticus & ATCC 17749 \\
$V$. cholerae & ATCC 14100 \\
$V$. harveyi & ATCC 43516 \\
$V$. mediterranei & ATCC 43341 \\
$V$. shilonii & ATCC BAA-91 \\
$V$. splendidus & MCCC 1A04096 \\
$V$. mimicus & MCCC1A02602 \\
$V$. ichthyoenteri & MCCC1A00057 \\
\hline
\end{tabular}

Table 2 Information of primers used in this study

\begin{tabular}{|c|c|c|c|c|c|c|c|c|}
\hline Name & Name & $\begin{array}{l}\text { Sequence } \\
\left(5^{\prime}-3^{\prime}\right)\end{array}$ & $\begin{array}{l}\text { Primer } \\
\text { length } \\
\text { (bp) }\end{array}$ & $\begin{array}{l}\text { Amplicon } \\
\text { size (bp) }\end{array}$ & Site & Site & Site & $\begin{array}{l}\text { GenBa } \\
\text { numbe }\end{array}$ \\
\hline$V P_{A H P N D^{-}}$ & $\mathrm{F}$ & $5 '-$ & 29 & 372 & 372 & 378. & $>\mathrm{NC}_{-}-$ & $>\mathrm{NC}_{-}-$ \\
\hline \multirow[t]{2}{*}{1} & & \multicolumn{4}{|c|}{ CATCTTTGACGGAATTTAACCCTAACAAT } & 406 & 025152.1 & 02515 \\
\hline & $\mathrm{R}$ & \multicolumn{4}{|c|}{ TAACTAAACCAATGTAATCATCTTTTGCCG } & $\begin{array}{l}749 . . \\
720\end{array}$ & & \\
\hline
\end{tabular}




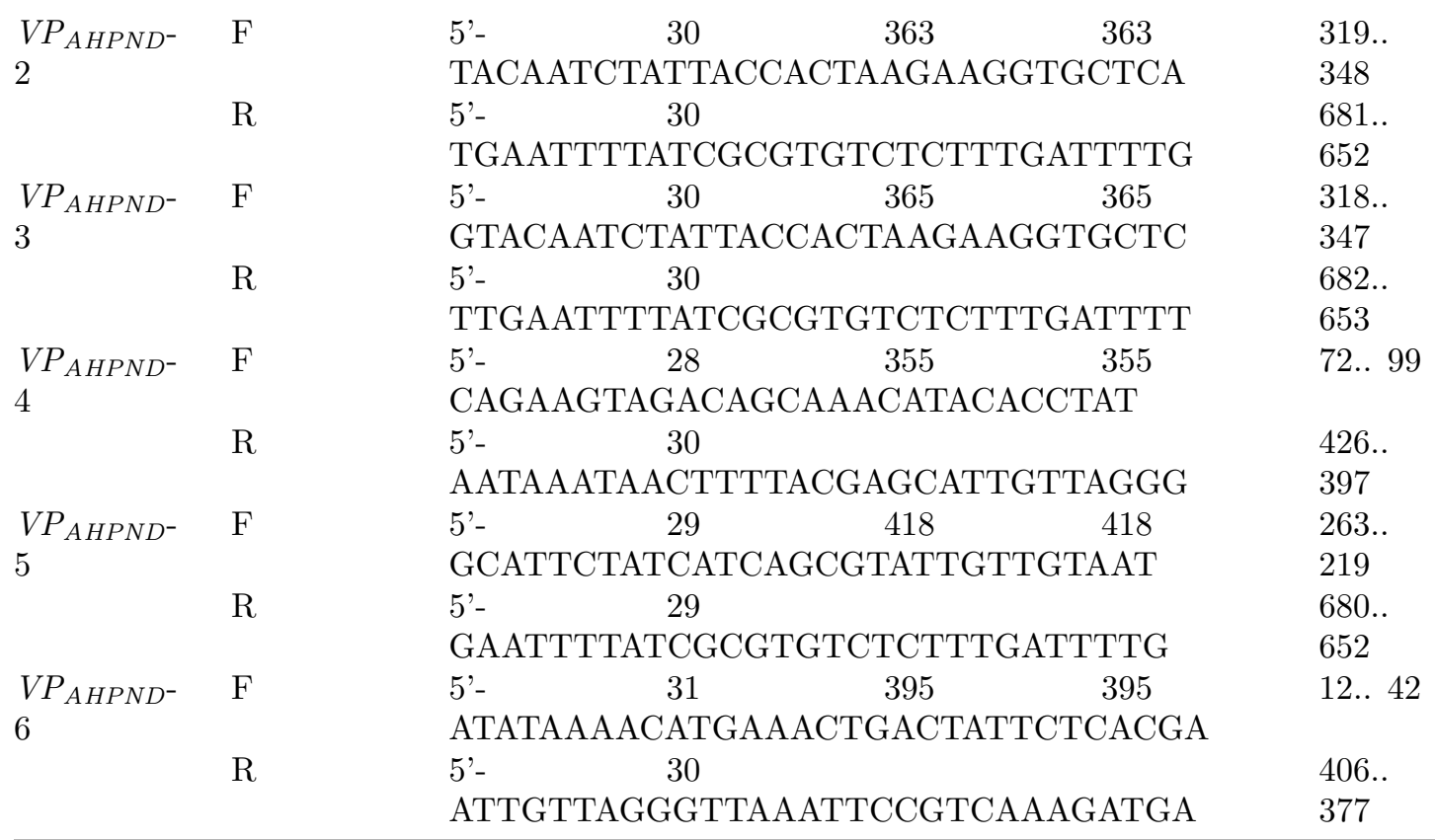

Table 3. Sequences of primer-probe sets and targeting areas

\begin{tabular}{lll}
\hline Name & Name & Sequence (5'-3') \\
$V P_{A H P N D^{-1}}$ & $\mathrm{~F}$ & 5'- CATCTTTGACGGAATTTAACCCTAACAAT \\
& $\mathrm{mR}$ & 5'- Biotin-TAACTAAACCAATGTAATCATCTTTTGCCG \\
& $\mathrm{P} 1$ & 5'-FITC-ATGAGCCAGATATTGAAAACATTTGGGAAC[THF]ATTACGTGACAGAAT - SpC3 \\
$V P_{A H P N D^{-3}}$ & $\mathrm{~F}$ & 5'- GTACAATCTATTACCACTAAGAAGGTGCTC \\
& $\mathrm{mR}$ & 5'- Biotin-TTGAATTTTATCGCGTGTCTCTTTGATTTT \\
& $\mathrm{P} 2$ & 5'-FITC-GCCAAATACGCCAAATGAGCCAGATATTGAAAACA[THF]TTGGGAACAA - SpC3 \\
\hline
\end{tabular}

Table 4. Mismatching of primer-probe

\begin{tabular}{ll}
\hline Name & Sequence (5'-3') \\
mR1 & 5'-Biotin-TAACTAAACCTATGTAATGATCTTTTG \\
mP2 & 5'-FITC- ATGAGCCAGCTATTGATAATATTTGGGAAC[THF]ATTACGTGACTGAAT- SpC3 \\
\hline
\end{tabular}

Table 5 Evaluation of the RPA-LFS in 65 collected shrimps and 10 seawater samples.

\begin{tabular}{|c|c|c|c|c|}
\hline Type & Number & The number of positive samples & The number of positive samples & The number of pos \\
\hline & & RPA-LFS & & AP4 \\
\hline shrimp & 65 & 20 & & 20 \\
\hline seawater & 10 & 3 & & 3 \\
\hline
\end{tabular}

\section{Figure legends}

Fig. 1. Agarose gel electrophoresis of six pairs primer of RPA assay result. Six pairs of primers targeting the PirAB gene were screened using RPA assay, and the amplification results are detected on the agarose 
gel. The name of each primer set is indicated at the top of the lane. The NTC lane represents no template control of the respective RPA reaction. The band sizes of the DNA ladder are shown on the right. The images represent results from three independent experiments.

Fig. 2. Agarose gel electrophoresis for the specificity verification of primer pairs $V P_{A H P N D}-1$ (Panel a ) and $V P_{A H P N D}-3$ (Panel $\left.\mathbf{b}\right)$. The detection specificity of each primer set is measured by RPA amplification using different bacterial genomic DNA and results are showed on the agarose gel images. Each species name of the bacterium is indicated on the top of each lane. The NTC lane is the no template control. The size of each band of the DNA ladder is indicated on the left of the gel image. The images represent results from three independent experiments.

Fig. 3. Screening of primer-probe sets for RPA-LFS. a. Test of un-mismatched primer-probe sets probe1/VP $A H P N D-1$ and probe2/VPAHPND -3 . b. Test of mismatched primer-probe sets mprobe1/VP $A H P N D$-1 was compared with unmodified primer-probe sets probe $1 / V P_{A H P N D}-1$. The amplification performance of primer-probe sets was evaluated by RPA amplification and LFS detection. The name of each primer-probe set is indicated on the top of the corresponding strip. The NTC lane is the no template control. The positions of test and control lines are marked on the right of the strip image. The image represents results from three independent experiments.

Fig. 4. Optimal reaction temperature (Panel a ) and time (Panel b ) of the RPA-LFS assay. Different reaction temperature ranging from $25^{\circ} \mathrm{C}$ to $45^{\circ} \mathrm{C}$ and reaction time varying from 5 min to 40 min are performed. Each temperature and time parameter are indicated on the top of the strip. The NTC strip is the no template control. The positions of test and control lines are marked on the right. The images represent results from three independent experiments.

Fig. 5.Exclusivity and inclusivity verification of the RPA-LFS assay. The exclusivity and inclusivity detection of RPA-LFS is performed using the genomic DNA of 10 other common vibriopathogens (Panel a ) and 10 other $V P_{A H P N D}$ isolated from shrimps infected with AHPND (Panel b ), respectively. The pathogenic vibrio parahaemolyticus $\left(V P_{A H P N D}\right)$ isolated from shrimp farms where AHPND occurred is used as the positive control. Each species name of the bacterium is indicated on the top of each strip. The NTC strip is the no template control. The positions of test and control lines are marked on the right of the strip image. The images represent results from three independent experiments.

Fig. 6. Detection limit of the RPA-LFS assay. The detection limited of the RPA-LFS assay is measured in not only the pure $V P_{A H P N D}$ culture (Panel a ) but also the interference with shrimp homogenate (Panel b ) with final concentrations ranging from $10^{7} \mathrm{CFU} / \mathrm{mL}$ to $10^{1} \mathrm{CFU} / \mathrm{mL}$. The amounts of templates are indicated on the top of each strip. The NTC strip is the no template control. The positions of test and control lines are marked on the right. The images represent results from three independent experiments.

Fig. 7. Detection limit of the RPA-LFS assay. The detection limit of the RPA-LFS assay is measured in not only the pure plasmid pVA1 (Panel a ) but also the interference with shrimp homogenate (Panel b ) with final concentrations ranging from $10^{7}$ copies $/ \mu \mathrm{L}$ to $10^{0}$ copies $/ \mu \mathrm{L}$. The amounts of templates are indicated on the top of each strip. The NTC strip is the no template control. The positions of test and control lines are marked on the right. The images represent results from three independent experiments.

Fig. 8. Detection of artificially spiked shrimps after enrichment using the RPA-LFS assay. Low concentration-spiked seafoods with $V P_{A H P N D}\left(10^{2} \mathrm{CFU} / 10 \mathrm{~g}^{\sim} 10^{0} \mathrm{CFU} / 10 \mathrm{~g}\right)$ are detected using RPA-LFS after enrichment for $0 \mathrm{~h}^{\sim} 24 \mathrm{~h}$. The enrichment time (in hours) is indicated on the top of each strip. The NTC strip is the no template control. The concentrations of spiked $V P_{A H P N D}$ is listed at the top of the image. The positions of test and control lines are marked on the right. The images represent results from three independent experiments. 

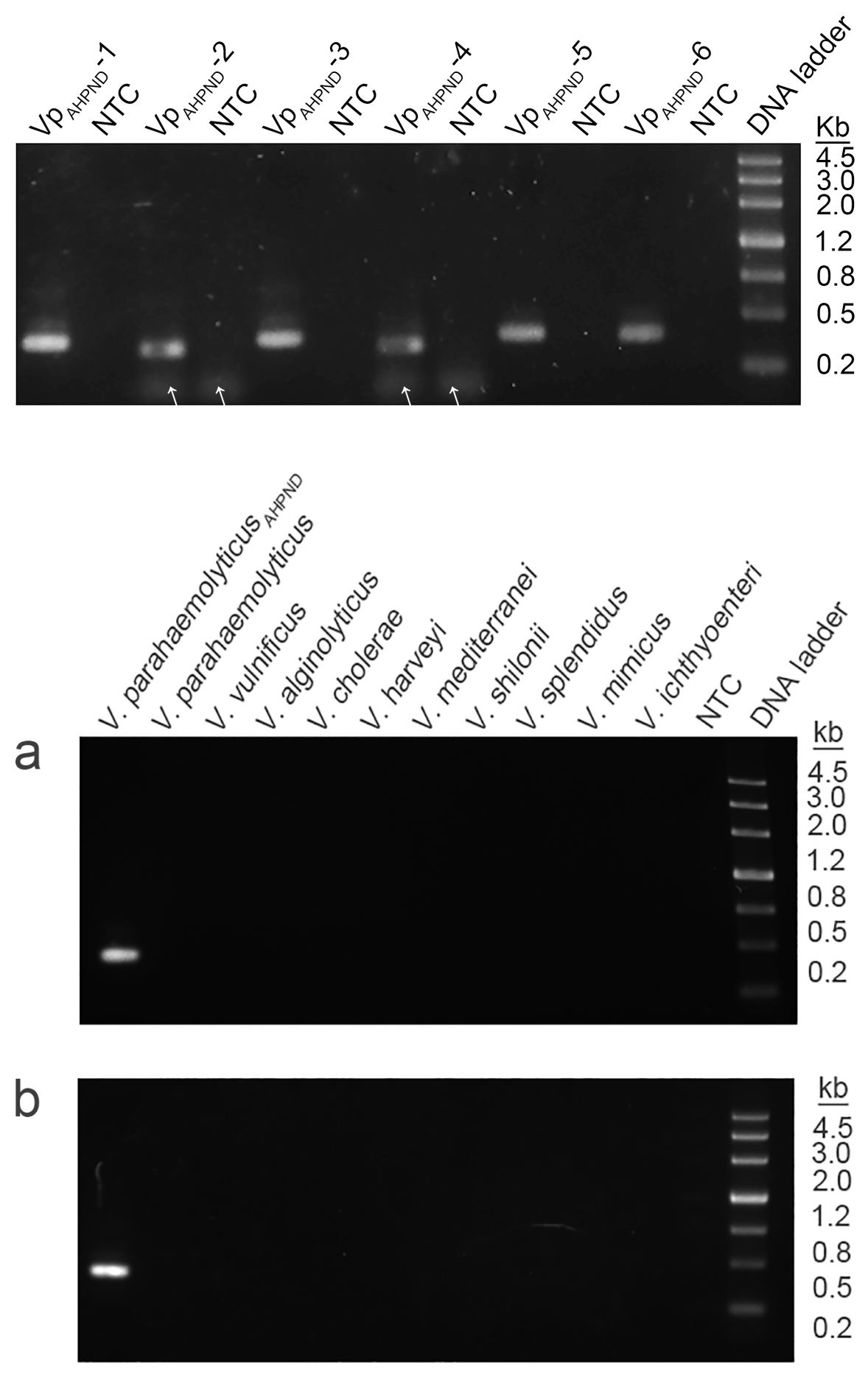


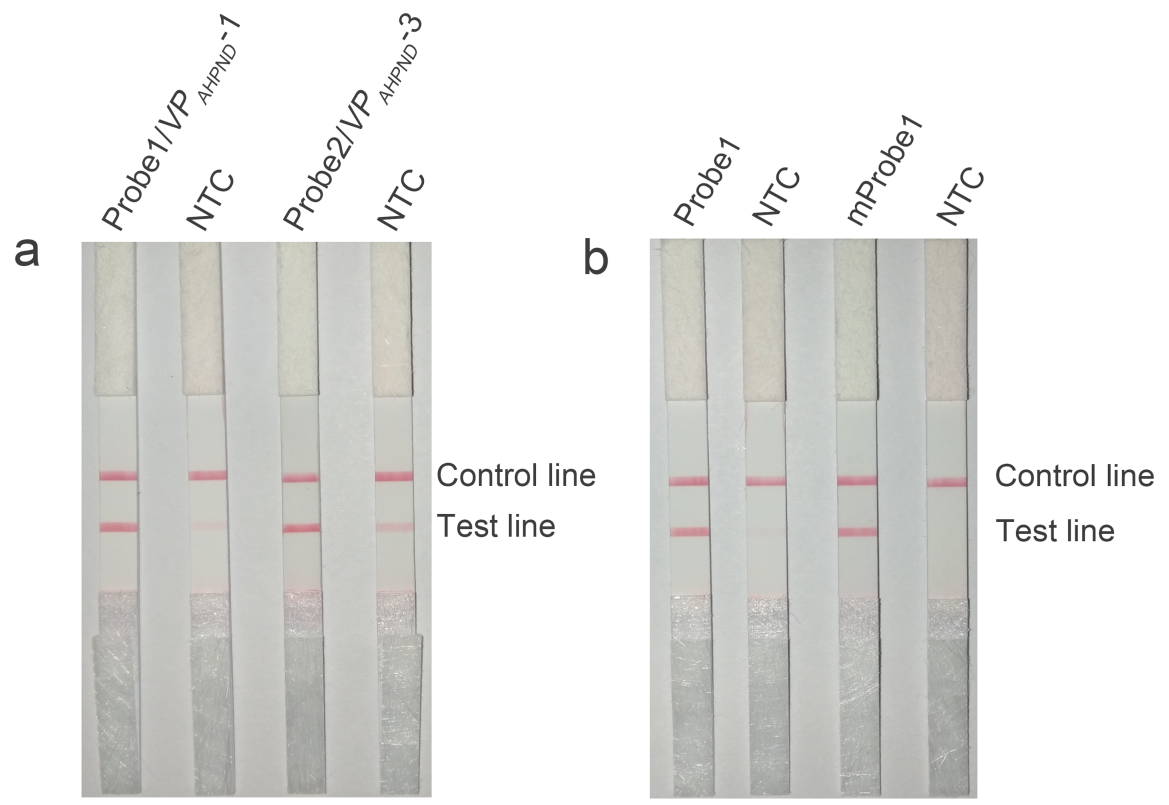

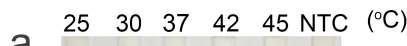
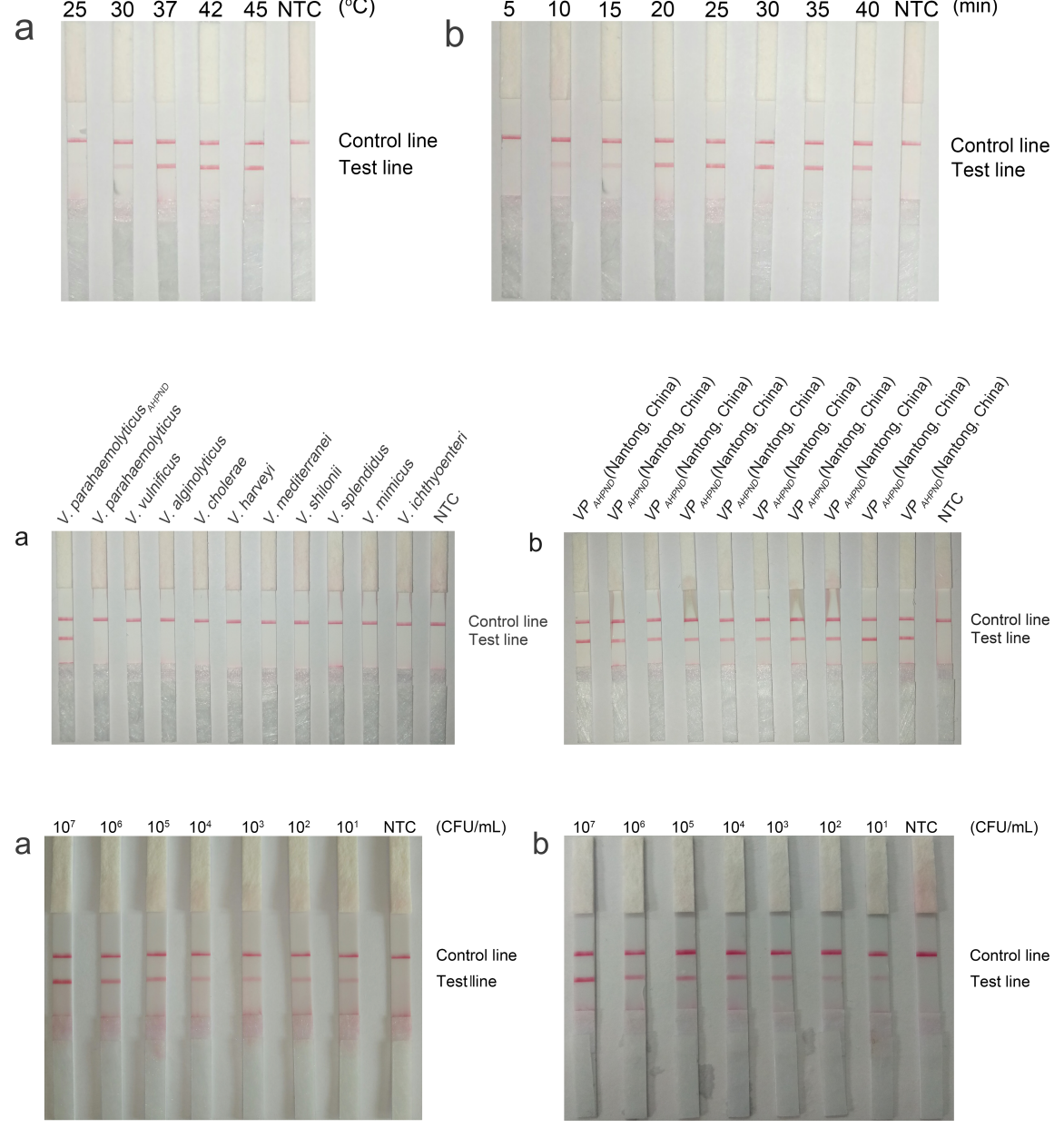
a

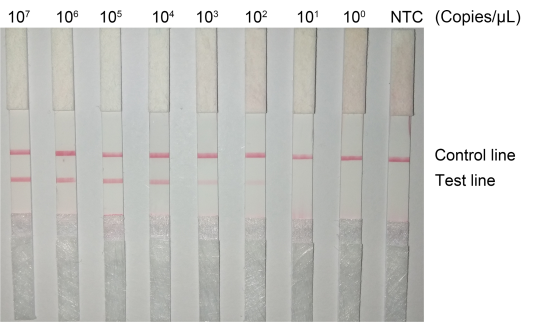

b

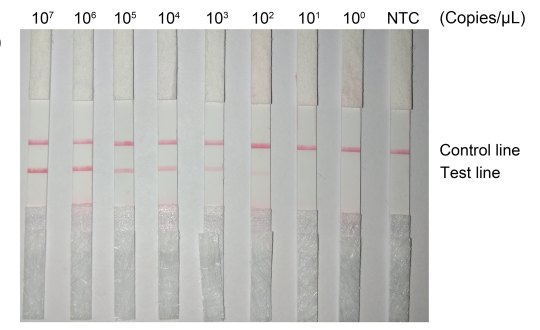

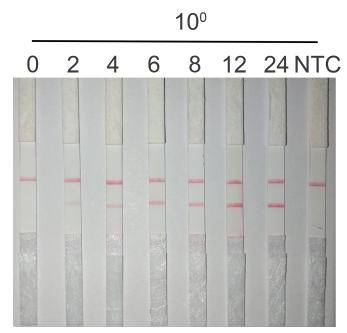
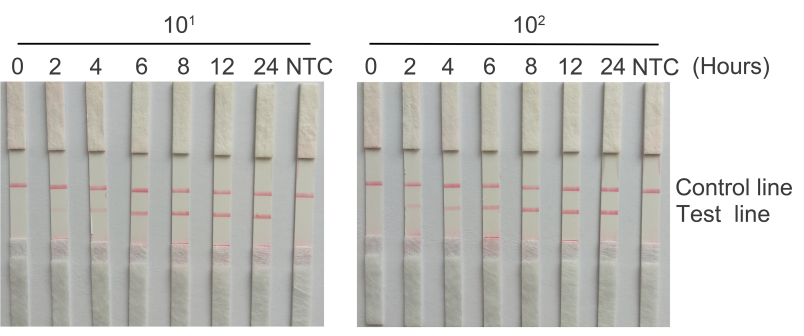